\title{
Velocity encoded cardiovascular magnetic resonance to assess left atrial appendage emptying
}

Kai Muellerleile ${ }^{1 *}$, Arian Sultan', Michael Groth², Daniel Steven', Boris Hoffmann', Gerhard Adam², Gunnar K Lund², Thomas Rostock ${ }^{1}$ and Stephan Willems ${ }^{1}$

\begin{abstract}
Background: The presence of impaired left atrial appendage (LAA) function identifies patients who are prone to thrombus formation in the LAA and therefore being at high risk for subsequent cardioembolic stroke. LAA function is typically assessed by measurements of LAA emptying velocities using transesophageal echocardiography (TEE) in clinical routine. This study aimed at evaluating the feasibility of assessing LAA emptying by velocity encoded (VENC) cardiovascular magnetic resonance (CMR).
\end{abstract}

Methods: This study included 30 patients with sinus rhythm $(n=18)$ or atrial fibrillation $(n=12)$. VENC-CMR velocity measurements were performed perpendicular to the orifice of the LAA. Peak velocities were measured of passive diastolic LAA emptying (e-wave) in all patients. Peak velocities of active, late-diastolic LAA emptying (a-wave) were assessed in patients with sinus rhythm. Correlation and agreement was analyzed between VENC-CMR and TEE measurements of e- and a-wave peak velocities.

Results: A significant correlation and good agreement was found between VENC-CMR and TEE measurements of maximal e-wave velocities $(r=0.61, P<0.001$; mean difference $0 \pm 10 \mathrm{~cm} / \mathrm{s})$. The a-wave was detectable by VENCCMR in all patients with sinus rhythm. Correlation was also significant for measurements of peak a-wave velocities between VENC-CMR and TEE $(r=0.71, P<0.001)$. There was no significant correlation of LAA emptying velocities with clinical characteristics and only a modest negative correlation of passive LAA emptying with LA function.

Conclusions: The assessment of active and passive LAA emptying by VENC-CMR is feasible. Further evaluation is required of potential future clinical applications such as risk stratification for cardioembolic stroke.

Keywords: Left atrial appendage, Atrial fibrillation, Stroke, Cardiovascular magnetic resonance, Transesophageal echocardiography.

\section{Background}

The left atrial appendage (LAA) is the major source of thrombotic material in patients with cardioembolic stroke [1]. Thrombus formation is promoted by an impaired LAA function with reduced emptying of the LAA [2]. An impaired LAA function is typically related to atrial fibrillation (AF). However, LAA function can also be reduced in patients with sinus rhythm (SR) due to transient stunning after cardioversion or sustained

\footnotetext{
*Correspondence: ka.muellerleile@uke.de

${ }^{1}$ Center for Cardiology and Cardiovascular Surgery, University Medical Center Hamburg-Eppendorf, Martinistrasse 52, D - 20246, Hamburg, Germany Full list of author information is available at the end of the article
}

dysfunction after catheter ablation of AF [3,4]. The assessment of LAA emptying aims at estimating the risk for thromboembolic events in several clinical settings and is typically performed by Doppler transesophageal echocardiography (TEE) [1,5]. However, TEE is invasive, requires sedation and is associated with the potential risk of complications in the upper gastro-intestinal-tract [6]. Therefore growing interest has been recently paid to non-invasive techniques for the assessment of LAA emptying [7].

Cardiovascular magnetic resonance (CMR) offers unique features in patients at risk for stroke: Apart from the ability to detect LAA thrombi and to measure LAA

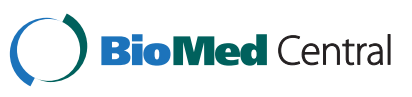


dimensions, LA fibrosis quantified by Late Gadolinium Enhancement (LGE) CMR was recently proposed as a novel marker for high-risk patients [8-11]. Furthermore, plaques with retrograde flow in the descending aorta can be assessed by CMR as a potential non-cardiac source for cerebral embolism [12,13]. Velocity-encoded (VENC) CMR serves as the reference method for the quantification of intracardiac flow, indicating a potential use for measurements of LAA flow as a component of a comprehensive CMR protocol in patients at risk for stroke [14]. However, the assessment of LAA function by CMR has not been studied yet. We hypothesized that VENCCMR could be used to assess LAA emptying velocities. Thus, this study compared measurements of LAA emptying velocities between VENC-CMR and TEE.

\section{Methods}

\section{Patients}

The study was approved by the local ethics committee and all patients gave their written informed consent. TEE and CMR were performed in 30 consecutive patients before scheduled catheter AF ablation for the assessment of LA and pulmonary venous anatomy. TEE was performed as a part of clinical routine to exclude LAA thrombi before AF ablation. CMR and TEE were carried out on the same day. Eighteen (60\%) patients with a history of paroxysmal AF were in SR and 12 (40\%) patients with persistent AF were in AF when CMR and TEE were performed. Patients were excluded if contraindications for CMR such as implanted pacemakers or severe claustrophobia were present.

\section{LAA emptying velocities by VENC-CMR}

CMR was performed using a 1.5 Tesla system (Achieva, Philips Medical Systems, Best, The Netherlands). All sequences were ECG-triggered and breath-held. Scout images were performed in axial, coronal and sagittal orientation. Standard retrospectively vector-ECG gated cine-CMR was performed in short- and long-axis to assess cardiac volumes and function. Additionally, cineCMR of the LAA was performed in a long-axis view (Figure 1A) and in the short axis orientation (Figure 1B). Typical imaging parameters of cine-CMR were as follows: pixel spacing $1.56 \times 1.56 \mathrm{~mm}^{2}$, slice thickness $8 \mathrm{~mm}$, time to repetition $=2.8 \mathrm{~ms}$, echo time $=1.4 \mathrm{~ms}$, flip angle $=60^{\circ}$. A breath-held, retrospectively vectorECG gated, two-dimensional flow-sensitive phasecontrast gradient-echo sequence was used for VENCCMR flow measurements perpendicular to the orifice of the LAA (Figure 1). Furthermore, VENC-CMR transmitral flow measurements were performed to facilitate the interpretation of LAA flow curves. Typical imaging parameters of VENC-CMR were as follows: Pixel spacing $1.37 \times 1.37 \mathrm{~mm}^{2}$, slice thickness $8 \mathrm{~mm}$, time to repetition $=4.5 \mathrm{~ms}$, echo time $=2.9 \mathrm{~ms}$, flip angle $=15^{\circ}$, 50 frames per RR-interval resulted in an effective temporal resolution of $22 \pm 8 \mathrm{~ms}$ ( $60 \%$ phase percentage), parallel imaging (SENSE reconstruction) with an acceleration factor of 2, encoding velocity was set to $100 \mathrm{~cm} / \mathrm{s}$, typical breathhold durations were about 25 seconds.

VENC- CMR data were analyzed using commercial software (ViewForum workstation R5.1, Philips Medical Systems, Best, The Netherlands). Peak velocities were measured of passive diastolic LAA emptying (e-wave) in all patients. Peak velocities of active, late-diastolic LAA emptying (a-wave) were assessed in patients with sinus rhythm. Figure 2 demonstrates measurements of LAA emptying velocities by VENC- CMR in patients with SR and in patients with AF. All data analyses were repeated with an interval of at least one week to assess intraobserver agreement. An additional reading was performed in all patients by a second observer to assess inter-observer agreement.

\section{LAA emptying velocities by TEE}

TEE was performed using a Vivid S6 system equipped with a multiplane $5.0 \mathrm{MHz}$ transducer (GE Vingmed Ultrasound, Horten, Norway) as a part of clinical routine to exclude LAA thrombi before AF ablation. All patients received a mild sedation with propofol and additional topical anesthesia using a lidocaine gel in agreement with current recommendations [15]. LAA views were obtained in transverse and longitudinal orientations. LAA flow was assessed using pulsed-wave Doppler by placing the sample volume within the orifice of the LAA (Figure 3). Measurements of five consecutive beats were averaged to define e-wave velocities in patients with $\mathrm{AF}$ [16].

\section{Statistical analysis}

Statistical analysis was performed using GraphPad Prism version 5.00 for Windows (GraphPad Software, San Diego, CA, USA). Continuous data are presented as median and interquartile range (IQR). Normality testing was performed using the D'Agostino-Pearson omnibus method. Bland-Altman analysis was used to assess agreement between observers and methods. Correlation between VENC- CMR and TEE was assessed using Pearson`s correlation coefficient. Statistical significance was set to $\mathrm{P}<0.05$.

\section{Results}

\section{General}

The major patient characteristics are provided in Table 1 , indicating a typical AF study population with enlarged LA volumes and preserved LV function (Table 1) [17]. No complications were observed during the CMR and the TEE procedures, respectively. None of the patients 

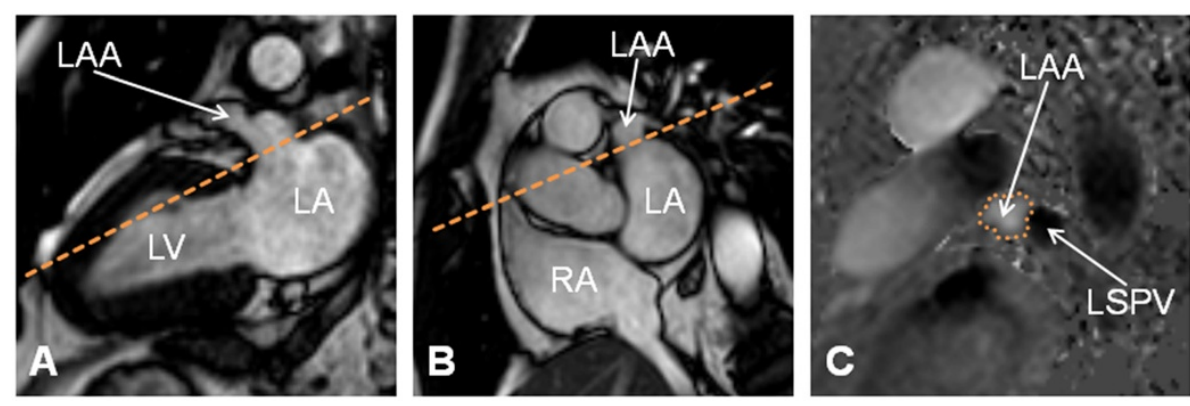

Figure 1 VENC-CMR slice positioning for LAA flow measurements. A VENC-CMR slice was positioned (dashed line) perpendicular to the LAA orifice on long-axis (Figure 1A) and short-axis (Figure 1B) cine-CMR views. The region of interest for flow measurements was drawn around the LAA orifice as outlined by the dotted line on the phase VENC-CMR image (Figure 1C). Abbreviations: LAA =left atrial appendage, LA =left atrium, $\mathrm{LV}=$ left ventricle, $\mathrm{RA}=$ right atrium, $\mathrm{LSPV}=$ left superior pulmonary vein

had evidence of LAA thrombus on CMR or TEE. All subsequent $\mathrm{AF}$ ablation procedures were performed without cerebral complications. VENC-CMR provided sufficient image quality to enable quantification of peak LAA e- and a-wave velocities in all patients. Analysis of VENC-CMR data was fast, requiring less than 5 minutes for complete post-processing and measurements. Median peak e-wave velocity by VENC-CMR was 22 (IQR $16-31) \mathrm{cm} / \mathrm{s}$ in the whole study population, 28 (IQR $20-42) \mathrm{cm} / \mathrm{s}$ in patients with AF and 18 (IQR 14-24) $\mathrm{cm} / \mathrm{s}$ in patients with SR, respectively. Median peak awave velocity by VENC-CMR was 47 (IQR 33-64) $\mathrm{cm} / \mathrm{s}$ in patients with SR.

\section{Reproducibility of VENC-CMR data analysis}

Intra- and inter-observer agreements were strong for data analysis of VENC-CMR peak e-wave velocities with a mean bias of $1 \pm 4$ and $2 \pm 4 \mathrm{~cm} / \mathrm{s}$, respectively. There were also strong intra- and inter-observer agreements for data analysis of VENC-CMR peak a-wave velocities with a mean bias of $0 \pm 3$ and $0 \pm 3 \mathrm{~cm} / \mathrm{s}$, respectively.

\section{VENC-CMR vs. TEE}

A significant correlation was found between VENCCMR and TEE measurements of peak e-wave velocities $(\mathrm{r}=0.61, \mathrm{P}<0.001)$ with a mean difference $0 \pm 10 \mathrm{~cm} / \mathrm{s}$ (Figure $4 \mathrm{~A}$ and $\mathrm{C}$ ) in the whole study population. No significant correlation was found between VENC-CMR and TEE in a separate analysis of patients with SR $(\mathrm{r}=0.33, \mathrm{P}=0.17)$ and $\mathrm{AF}(\mathrm{r}=0.58, \mathrm{P}=0.06)$, respectively. VENC-CMR permitted identification of the a-wave in all patients with sinus rhythm. Correlation was also significant for measurements of peak a-wave velocities between VENC-CMR and TEE $(\mathrm{r}=0.71, \mathrm{P}<0.001$, Figure 4B). However, a mean difference of $10 \pm 15 \mathrm{~cm} / \mathrm{s}$ $(\mathrm{P}<0.05)$ was found between VENC-CMR and TEE peak a-wave velocities, indicating a potential systematic underestimation of peak a-wave velocities by VENCCMR (Figure 4D).

\section{LAA emptying vs. Clinical and CMR parameters}

There was no significant correlation of VENC-CMR peak $\mathrm{e}$ - and a-wave velocities with heart rate $(\mathrm{r}=0.31$ and $r=0.18)$, body-mass-index $(r=-0.01$ and $r=0.26)$,
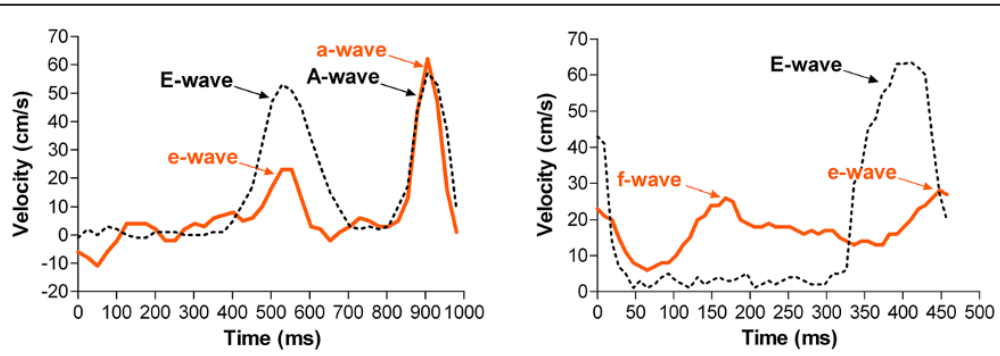

Figure 2 VENC-CMR measurements of LAA emptying velocities. LAA flow velocity profiles by VENC-CMR in a patient with SR (Figure 2A) and in a patient with AF (Figure 2B). Transmitral flow velocity profiles (black dotted lines) were superimposed to enable a clear identification of e- and a-waves, respectively. The e-wave was defined by the coincidence with early, passive LA emptying (E-wave, Figure 2A). The a-wave was defined by the coincidence with late, active LA emptying (A-wave, Figure 2A) in patients with SR. Peak e-wave velocity was $23 \mathrm{~cm} / \mathrm{s}$ and peak a-wave velocity was $62 \mathrm{~cm} / \mathrm{s}$ in patient A. In patients with AF, a temporal shift between transmitral E-wave and LAA e-wave was observed, that could possibly be related to RR-interval variability or irregular electrical LAA activation. The LAA e-waves were therefore differentiated from $\mathrm{f}$-waves by proximity rather than coincidence to passive diastolic LA emptying (E-wave) in patients with AF (Figure 2B). Peak e-wave velocity was $28 \mathrm{~cm} / \mathrm{s}$ in patient B. A prominent systolic f-wave was detected in this patient, related to electric activation of the LAA in AF. 


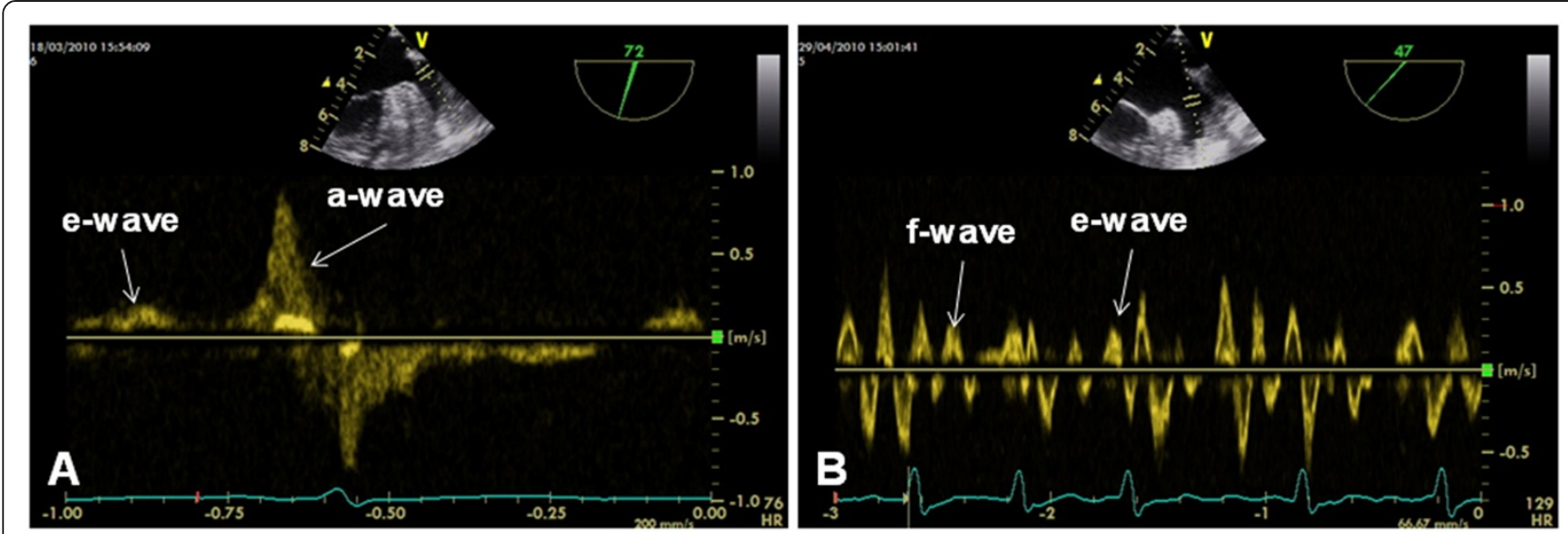

Figure 3 TEE measurements of LAA emptying velocities. LAA flow velocity profiles by TEE in a patient with SR (Figure 3A) and in a patient with AF (Figure 3B). Peak e-wave velocity was $28 \mathrm{~cm} / \mathrm{s}$ and peak a-wave velocity was $73 \mathrm{~cm} / \mathrm{s}$ in patient A. Mean peak e-wave velocity of five consecutive beats was $26 \mathrm{~cm} / \mathrm{s}$ in patient B. Prominent f-waves were detected similar to the VENC-CMR flow curve (Figure 3B), corresponding to high-amplitude atrial fibrillation waves in ECG in this patient.

systolic $(\mathrm{r}=-0.01$ and $\mathrm{r}=0.06)$ and diastolic $(\mathrm{r}=0.25$ and $\mathrm{r}=0.21)$ blood pressure, respectively $(\mathrm{P}=\mathrm{ns})$. Furthermore, there was no significant correlation of VENCCMR peak e- and a-wave velocities with $\mathrm{LV}$ end-diastolic $(r=0.26$ and $r=0.03)$, LV end-systolic $(r=0.25$ and $\mathrm{r}=-0.10)$ and LA $(\mathrm{r}=0.20$ and $\mathrm{r}=-0.21)$ volume indices or $\mathrm{LV}$ ejection fraction $(\mathrm{r}=-0.14$ and $\mathrm{r}=0.26)$. There was no significant correlation between peak LAA e-wave and mitral valve $E$-wave velocities $(r=0.31, P=0.15)$ as well as between peak LAA a-wave and mitral valve A-wave velocities $(\mathrm{r}=0.23, \mathrm{P}=0.44)$. A modest negative correlation was found between peak LAA e-wave velocities and LA ejection fraction $(r=-0.46, P<0.05)$ but not between peak LAA a-wave velocities and LA ejection fraction $(\mathrm{r}=-0.01, \mathrm{P}=0.99)$.

\section{Table 1 Patient characteristics}

\begin{tabular}{ll}
\hline Age (years) & $66(54-72)$ \\
Female sex & $10(33)$ \\
BMI & $28(24-30)$ \\
SR & $18(60)$ \\
AF & $12(40)$ \\
Ventricular heart rate $(\mathbf{b p m})$ & $69(58-78)$ \\
Systolic blood pressure $(\mathbf{m m H g})$ & $140(128-150)$ \\
Diastolic blood pressure $(\mathbf{m m H g})$ & $80(75-88)$ \\
LAVi $\left(\mathbf{m l} / \mathbf{m}^{2}\right)$ & $58(46-73)$ \\
LAEF $(\%)$ & $51(26-64)$ \\
LVEDVi $\left(\mathbf{m l} / \mathbf{m}^{\mathbf{2}}\right)$ & $75(62-81)$ \\
LVESVi $\left(\mathbf{m l} / \mathbf{m}^{2}\right)$ & $31(27-41)$ \\
LVEF $(\%)$ & $55(47-78)$ \\
\hline
\end{tabular}

\section{Discussion}

This study compared measurements of LAA emptying velocities between VENC-CMR and TEE in thirty consecutive patients. The major findings were:

- Inter- and intra-observer agreement was strong for data analysis of VENC-CMR LAA emptying velocity measurements.

- There was a significant correlation for measurements of peak LAA e- and a-wave velocities between VENC-CMR and TEE.

- There was no significant correlation of LAA emptying velocities with clinical characteristics and only a modest negative correlation of passive LAA emptying with LA function.

\section{Passive LAA emptying}

Passive LAA emptying is primarily related to a suction effect of passive LA emptying in early diastole in SR as well as in AF $[13,14]$. In SR, the e-wave velocity is a surrogate parameter for passive LAA emptying and is typically lower than active emptying velocities (Figure 3A) [14]. Being of little relevance in SR under physiological conditions, passive LAA emptying gains importance in situations with impaired active LAA emptying such as atrial stunning $[3,4,13,14]$. In patients with AF, LAA emptying is inherently passive. Doppler TEE differentiates variable systolic $\mathrm{f}$-waves related to electrical LAA activation from diastolic e-waves related to passive LA emptying, similar to e-waves in SR $[16,18]$. Principle methodological differences between VENC-CMR and TEE have to be considered for interpreting LAA flow curves in AF: CMR data acquisition is distributed to 

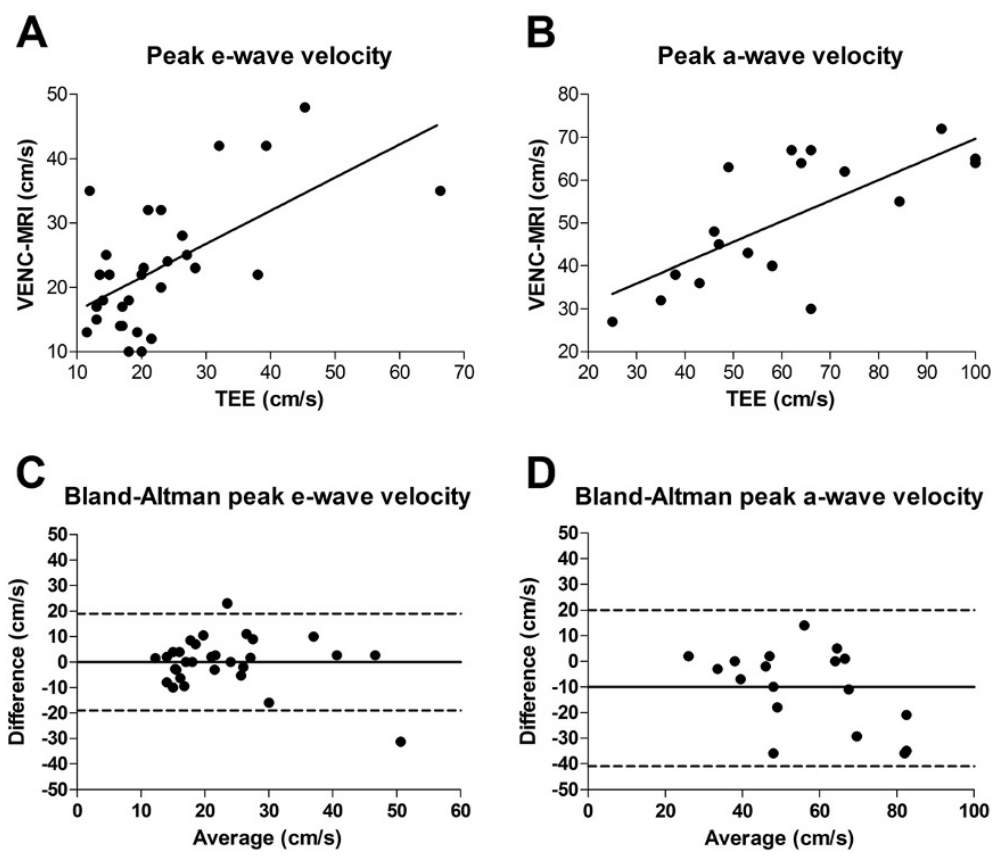

Figure 4 VENC-CMR vs. TEE. Figure 4A and C: Correlation and agreement between VENC-CMR and TEE measurements of peak e-wave velocities $(r=0.61, P<0.001$, mean difference $0 \pm 10 \mathrm{~cm} / \mathrm{s})$. Figure 4B: Correlation for measurements of peak a-wave velocities between VENC-CMR and TEE $(r=0.71, P<0.001)$. Figure 4D: Mean underestimation of $10 \pm 15 \mathrm{~cm} / \mathrm{s}$ for peak a-wave velocities $(P<0.05)$ by VENC-CMR compared to TEE. On Bland-Altman plots, middle continuous line indicates mean bias, and dashed lines above and below indicate 95\% confidence intervals.

several cardiac cycles in segmented ECG-gated sequences. Thus, LAA flow curves by VENC-CMR represent composed data from several cardiac cycles with variable RR-intervals and degrees of electrical LAA activation. We defined VENC-CMR e-waves in AF by proximity with superimposed passive LA emptying to facilitate a clear differentiation from systolic $f$-waves (Figures 2 and 3). Nevertheless, it is important to highlight the inherent difference between distinct $e$ - and fwaves in TEE compared to the averaged velocity peaks in VENC-CMR curves despite a similar appearance.

Despite the technically related differences, our findings indicate that VENC-CMR measurements of passive LAA are feasible. However, the non-significant correlation between CMR and TEE measurements within the subgroups of patient with $\mathrm{SR}$ and $\mathrm{AF}$ requires further evaluation in larger populations. Nevertheless, recent data indicate a potential role for CMR measurements of LAA dimensions and function in patients at risk for embolic stroke. Beinart et al. reported an independent predictive value of LAA dimensions for embolic cerebral events in a study with 144 patients [10]. Considering the well established association between reduced LAA emptying velocities and thrombus formation in the LAA, VENC-CMR could be an attractive adjunctive non-invasive tool in this context $[1,2]$. A comprehensive CMR protocol including measurements of LA fibrosis,
LAA dimensions and passive LAA emptying velocities could potentially improve risk stratification in patients with AF [8-11]. Furthermore, Donal et al. reported a high specificity of e-wave velocities by TEE during AF to predict recovery of active LAA emptying after AF ablation [19]. Thus, VENC-CMR measurements of passive LAA velocities could also be used for the non-invasive prediction of potential recovery of LAA contractility after successful AF ablation.

\section{Active LAA emptying}

Active LAA emptying is related to LAA contraction following the electric activation of the LA and LAA in SR. The LAA a-wave occurs simultaneously with the A-wave of transmitral flow immediately after the surface ECG $\mathrm{P}$-wave. LAA a-wave velocity is a surrogate parameter for active LAA emptying $[16,18]$. A reduced a-wave velocity can be present in SR as transient stunning after cardioversion or AF ablation, but is also related to ageing $[3,13,16,20]$. However, since TEE is an invasive procedure, data are limited on time course, potential predictors and modulators of LAA stunning after catheter ablation of AF $[16,18]$. Of particular interest are patients after ablation of persistent AF. In these patients, extended ablation strategies are necessary, which have the potential risk of an inadvertent electric isolation of the LAA [4,21]. Thus, there is a need for a 
non-invasive diagnostic tool permitting the proof of active LAA emptying after persistent AF ablation.

We found a significant correlation of a-wave velocities between VENC-CMR and TEE, indicating the feasibility of LAA emptying measurements using VENC-CMR. However, there was a mean underestimation of $10 \pm 15 \mathrm{~cm} / \mathrm{s}$ for peak a-wave velocities by VENC-CMR compared to TEE. This finding is in agreement with recent data on underestimation of vascular and valvular peak velocities by VENC-CMR compared to ultrasound measurements, upcoming advanced VENC sequences may overcome this discrepancy in future [22]. Nevertheless, VENC-CMR measurements appear to be potentially useful to differentiate low-risk patients with fully recovered active LAA emptying from high-risk patients with permanently missing active LAA emptying. Furthermore, VENC-CMR could be used in combination with LGE CMR to longitudinally monitor recovery of active LAA emptying and its relationship to the extent of ablation lesions $[23,24]$.

\section{LAA emptying and clinical characteristics}

We did not find a significant correlation between passive LAA emptying velocities and clinical parameters such as heart rate, body-mass-index, blood-pressure, LV volumes and function. Furthermore, LAA emptying velocities were only weakly associated with LA function. These findings are in agreement with recent data from TEE, underscoring that LAA emptying cannot be predicted by clinical characteristics or by assessing LA function $[16,18]$. Thus, LA and LAA function require separate assessment; VENC-CMR seems to be a promising tool in this context.

In summary, our study demonstrated the feasibility of non-invasive LAA emptying velocity measurements by VENC-CMR. Nevertheless, some principle technical differences between VENC-CMR and TEE have to be considered: Accurate VENC-CMR measurements require blood flow perpendicular to slice orientation, whereas Doppler TEE measurements depend on the correct alignment of probe orientation with blood flow. Thus, differences in sampling locations and slice/probe orientation with respect to LAA blood flow could have influenced the agreement between VENC-CMR and TEE measurements. VENC-CMR has the principle advantage of free slice orientation compared to the limited TTE acoustic window. In contrast, the superior temporal and spatial resolution constitutes a potential advantage of TEE. Furthermore, transthoracic echocardiography (TTE) was recently introduced as another non-invasive alternative to TEE for the assessment of LAA emptying [7].

However, the true incremental value of VENC-CMR compared to other techniques is the combinability of
LAA emptying measurements with novel CMR features such as the assessment of LA fibrosis [11]. VENC-CMR emptying measurements require one extra breathhold, adding less than one minute to a standard CMR protocol. Thus, there are several potential future applications of VENC-CMR LAA emptying measurements:

First, VENC-CMR LAA flow measurements could be integrated into a comprehensive CMR protocol for the evaluation of patients at risk for stroke. Such a protocol could incorporate information on LA dimensions and function, LAA emptying, LA fibrosis as well as on aortic plaque morphology and flow characteristics to improve current risk stratification for stroke [8-13]. Second, VENC-CMR LAA flow measurements could be combined with MR angiographies of the LA and pulmonary veins that are frequently performed in clinical routine before AF ablation procedures [25]. In this context, LAA flow measurements could be helpful to predict recovery of LAA function after successful AF ablation. Third, VENC-CMR LAA flow measurements could be used in combination with LGE CMR to assess LAA dysfunction and its association with LA ablation lesions after AF ablation. Furthermore, VENC-CMR LAA flow measurements could be helpful in patients who are candidates for percutaneous LAA closure procedures to simultaneously assess LAA morphology and function [26]. However, further evaluation of VENC-CMR LAA flow measurements is required in larger populations before clinical implementation.

\section{Limitations}

This feasibility study is mainly limited by the small study population. Furthermore, recent findings from transmitral flow measurements indicate an improved accuracy of intracardiac flow-measurements using threedimensional VENC-CMR sequences [27]. The use of a conventional two-dimensional VENC-CMR sequence constitutes a potential limitation of this study. In addition, future studies on VENC-CMR LAA flow measurements should address not only reproducibility of data analysis but also reproducibility of data acquisition. Nevertheless, our findings clearly demonstrated the feasibility of LAA emptying measurements by VENCCMR.

\section{Conclusions}

The assessment of active and passive LAA emptying by VENC-CMR is feasible. Thus, VENC-CMR has the potential to non-invasively identify patients with impaired LAA function who are at risk for stroke.

\section{Abbreviations}

BMI: Body-mass-index; SR: Sinus rhythm; AF: Atrial fibrillation; Bpm: Beats per minute; LAVi: Left atrial volume index; LVEDVi: Left ventricular end-diastolic volume index; LVESVi: Left ventricular end-systolic volume index; LAEF: Left 
atrial ejection fraction; LVEF: Left ventricular ejection fraction; : Numbers are median (interquartile range) for continuous and $\mathrm{n}$ (\% of total column number) for categorical data.

\section{Competing interests}

The authors declare that they have no competing interests.

\section{Authors' contributions}

All authors participated in editing and drafting of the manuscript. KM, AS, MG, GA, TR and SW contributed to concept, design and coordination of the present study. KM, MG, GKL and GA were involved in CMR data acquisition, analysis and interpretation. $\mathrm{KM}, \mathrm{AS}$, DS and BH were involved in TEE data acquisition, analysis and interpretation. All authors read and approved the final manuscript.

\section{Acknowledgements}

Parts of this manuscript were presented at the 2011 SCMR/EuroCMR Joint Session, February 3-6, Nice, France.

\section{Author details}

${ }^{1}$ Center for Cardiology and Cardiovascular Surgery, University Medical Center Hamburg-Eppendorf, Martinistrasse 52, D - 20246, Hamburg, Germany. ${ }^{2}$ Department of Diagnostic and Interventional Radiology, University Medical Center Hamburg-Eppendorf, Martinistrasse 52, D - 20246, Hamburg, Germany.

Received: 24 January 2012 Accepted: 3 May 2012 Published: 21 June 2012

\section{References}

1. Zabalgoitia M, Halperin JL, Pearce LA, Blackshear JL, Asinger RW, Hart RG: Transesophageal echocardiographic correlates of clinical risk of thromboembolism in nonvalvular atrial fibrillation. Stroke Prevention in Atrial Fibrillation III Investigators. J Am Coll Cardiol 1998, 31:1622-1626.

2. Kaneko K, Hirono O, Fatema K, Zhang X, Takeishi Y, Kayama T, Kubota I: Direct evidence that sustained dysfunction of left atrial appendage contributes to the occurrence of cardiogenic brain embolism in patients with paroxysmal atrial fibrillation. Intern Med 2003, 42:1077-1083.

3. Fatkin D, Kuchar DL, Thorburn CW, Feneley MP: Transesophageal echocardiography before and during direct current cardioversion of atrial fibrillation: evidence for "atrial stunning" as a mechanism of thromboembolic complications. J Am Coll Cardiol 1994, 23:307-316.

4. Chan CP, Wong WS, Pumprueg S, Veerareddy S, Billakanty S, Ellis C, Chae S, Buerkel D, Aasbo J, Crawford T, et al.: Inadvertent electrical isolation of the left atrial appendage during catheter ablation of persistent atrial fibrillation. Hear Rhythm 2010, 7:173-180.

5. Fuster V, Ryden LE, Cannom DS, Crijns HJ, Curtis AB, Ellenbogen KA, Halperin JL, Le Heuzey JY, Kay GN, Lowe JE, et al.: ACC/AHA/ESC 2006 Guidelines for the Management of Patients with Atrial Fibrillation: a report of the American College of Cardiology/American Heart Association Task Force on Practice Guidelines and the European Society of Cardiology Committee for Practice Guidelines (Writing Committee to Revise the 2001 Guidelines for the Management of Patients With Atrial Fibrillation): developed in collaboration with the European Heart Rhythm Association and the Heart Rhythm Society. Circulation 2006, 114:e257-354

6. Hilberath JN, Oakes DA, Shernan SK, Bulwer BE, D'Ambra MN, Eltzschig HK: Safety of transesophageal echocardiography. J Am Soc Echocardiogr 2010, 23:1115-1127. quiz 1220-1111.

7. Uretsky S, Shah A, Bangalore S, Rosenberg L, Sarji R, Cantales DR, MacmillanMarotti D, Chaudhry FA, Sherrid MV: Assessment of left atrial appendage function with transthoracic tissue Doppler echocardiography. Eur J Echocardiogr 2009, 10:363-371.

8. Ohyama H, Hosomi N, Takahashi T, Mizushige K, Osaka K, Kohno M, Koziol $J \mathrm{~A}$ : Comparison of magnetic resonance imaging and transesophageal echocardiography in detection of thrombus in the left atrial appendage. Stroke 2003, 34:2436-2439.

9. Heist EK, Refaat M, Danik SB, Holmvang G, Ruskin JN, Mansour M: Analysis of the left atrial appendage by magnetic resonance angiography in patients with atrial fibrillation. Hear Rhythm 2006, 3:1313-1318.
10. Beinart R, Heist EK, Newell JB, Holmvang G, Ruskin JN, Mansour M: Left atrial appendage dimensions predict the risk of stroke/TIA in patients with atrial fibrillation. J Cardiovasc Electrophysiol 2010, 22:10-15.

11. Daccarett M, Badger TJ, Akoum N, Burgon NS, Mahnkopf C, Vergara G, Kholmovski E, McGann CJ, Parker D, Brachmann J, et al.: Association of left atrial fibrosis detected by delayed-enhancement magnetic resonance imaging and the risk of stroke in patients with atrial fibrillation. J Am Coll Cardiol 2011, 57:831-838.

12. Harloff A, Strecker C, Dudler P, Nussbaumer A, Frydrychowicz A, Olschewski M, Bock J, Stalder AF, Stroh AL, Weiller C, et al.: Retrograde embolism from the descending aorta: visualization by multidirectional 3D velocity mapping in cryptogenic stroke. Stroke 2009, 40:1505-1508.

13. Harloff A, Simon J, Brendecke S, Assefa D, Helbing T, Frydrychowicz A, Weber J, Olschewski M, Strecker C, Hennig J, et al.: Complex plaques in the proximal descending aorta: an underestimated embolic source of stroke. Stroke 2010, 41:1145-1150.

14. Marsan NA, Westenberg JJ, Ypenburg C, Delgado V, van Bommel RJ, Roes SD, Nucifora G, van der Geest RJ, de Roos A, Reiber JC, et al.: Quantification of functional mitral regurgitation by real-time 3D echocardiography: comparison with 3D velocity-encoded cardiac magnetic resonance. JACC Cardiovasc Imaging 2009, 2:1245-1252.

15. Flachskampf FA, Badano L, Daniel WG, Feneck RO, Fox KF, Fraser AG, Pasquet A, Pepi M, Perez de Isla L, Zamorano JL, et al.: Recommendations for transoesophageal echocardiography: update 2010. Eur J Echocardiogr 2010, 11:557-576.

16. Donal E, Yamada H, Leclercq C, Herpin D: The left atrial appendage, a small, blind-ended structure: a review of its echocardiographic evaluation and its clinical role. Chest 2005, 128:1853-1862.

17. Maceira AM, Cosin-Sales J, Roughton M, Prasad SK, Pennell DJ: Reference left atrial dimensions and volumes by steady state free precession cardiovascular magnetic resonance. J Cardiovasc Magn Reson 2010, 12:65.

18. Agmon Y, Khandheria BK, Gentile F, Seward JB: Echocardiographic assessment of the left atrial appendage. J Am Coll Cardiol 1999, 34:1867-1877

19. Donal E, Grimm RA, Yamada H, Kim YJ, Marrouche N, Natale A, Thomas JD: Usefulness of Doppler assessment of pulmonary vein and left atrial appendage flow following pulmonary vein isolation of chronic atrial fibrillation in predicting recovery of left atrial function. Am J Cardio/ 2005, 95:941-947.

20. Agmon Y, Khandheria BK, Meissner I, Schwartz GL, Petterson TM, O'Fallon WM, Gentile F, Whisnant JP, Wiebers DO, Covalt JL, Seward JB: Left atrial appendage flow velocities in subjects with normal left ventricular function. Am J Cardiol 2000, 86:769-773.

21. Rostock T, Steven D, Hoffmann B, Servatius H, Drewitz I, Sydow K, Mullerleile K, Ventura R, Wegscheider K, Meinertz T, Willems S: Chronic atrial fibrillation is a biatrial arrhythmia: data from catheter ablation of chronic atrial fibrillation aiming arrhythmia termination using a sequential ablation approach. Circ Arrhythm Electrophysiol 2008, 1:344-353.

22. Baltes C, Hansen MS, Tsao J, Kozerke S, Rezavi R, Pedersen EM, Boesiger P: Determination of peak velocity in stenotic areas: echocardiography versus k-t SENSE accelerated MR Fourier velocity encoding. Radiology 2008, 246:249-257.

23. Peters DC, Wylie JV, Hauser TH, Kissinger KV, Botnar RM, Essebag V Josephson ME, Manning WJ: Detection of pulmonary vein and left atrial scar after catheter ablation with three-dimensional navigator-gated delayed enhancement MR imaging: initial experience. Radiology 2007, 243:690-695.

24. McGann CJ, Kholmovski EG, Oakes RS, Blauer JJ, Daccarett M, Segerson N, Airey KJ, Akoum N, Fish E, Badger TJ, et al.: New magnetic resonance imaging-based method for defining the extent of left atrial wall injury after the ablation of atrial fibrillation. J Am Coll Cardiol 2008, 52:1263-1271

25. Hendel RC, Patel MR, Kramer CM, Poon M, Carr JC, Gerstad NA, Gillam LD, Hodgson JM, Kim RJ, Lesser JR, et al.: ACCF/ACR/SCCT/SCMR/ASNC/NASCI/ SCAI/SIR 2006 appropriateness criteria for cardiac computed tomography and cardiac magnetic resonance imaging: a report of the American College of Cardiology Foundation Quality Strategic Directions Committee Appropriateness Criteria Working Group, American College of Radiology, Society of Cardiovascular Computed Tomography, Society for Cardiovascular Magnetic Resonance, American Society of Nuclear Cardiology, North American Society for Cardiac Imaging, Society for 
Cardiovascular Angiography and Interventions, and Society of Interventional Radiology. J Am Coll Cardiol 2006, 48:1475-1497.

26. Mohrs OK, Wunderlich N, Petersen SE, Pottmeyer A, Kauczor HU: Contrastenhanced CMR in patients after percutaneous closure of the left atrial appendage: a pilot study. J Cardiovasc Magn Reson 2011, 13:33.

27. Westenberg JJ, Roes SD, Ajmone Marsan N, Binnendijk NM, Doornbos J, Bax $J$ J, Reiber JH, de Roos A, van der Geest RJ: Mitral valve and tricuspid valve blood flow: accurate quantification with 3D velocity-encoded MR imaging with retrospective valve tracking. Radiology 2008, 249:792-800.

doi:10.1186/1532-429X-14-39

Cite this article as: Muellerleile et al:: Velocity encoded cardiovascular magnetic resonance to assess left atrial appendage emptying. Journal of Cardiovascular Magnetic Resonance 2012 14:39.

\section{Submit your next manuscript to BioMed Central and take full advantage of:}

- Convenient online submission

- Thorough peer review

- No space constraints or color figure charges

- Immediate publication on acceptance

- Inclusion in PubMed, CAS, Scopus and Google Scholar

- Research which is freely available for redistribution 